\title{
Edema pulmonar agudo por hidroclorotiazida: una reacción adversa poco conocida
}

\author{
PENNY YEN LEE*, MAURICIO SALINAS F***, PILAR LORA L.***, \\ ENRIQUE REYNOLDS H.*** y GIOVANNI ENCISO G.***
}

\section{Hydrochlorothiazide-induced acute pulmonary edema: a rare adverse reaction}

In Chile, hydrochlorothiazide is frequently prescribed as first line antihypertensive therapy. Among it's well known adverse reactions are: electrolytic disorders, hyperuricemia, dyslipidemia, agranulocytosis and azotemia. Acute pulmonary edema is a rare and potentially lethal adverse effect. Only 50 cases have been reported since 1968. In this article, we discuss a case of a 70 year old woman who, one hour after the ingestion of hydrochlorotiazide, presented acute and progressive dyspnea. Her clinical and radiologic findings are compatible with non-cardiogenic acute pulmonary edema.

Key words: Acute pulmonary edema, hidrochlorothiazide, drugs side effects, intensive care unit.

\section{Resumen}

En Chile, la hidroclorotiazida se utiliza ampliamente como terapia de primera línea en la hipertensión arterial esencial. Entre los efectos adversos más conocidos destacan: trastornos hidroelectrolíticos, hiperuricemia, dislipidemia, azotemia, entre otros. El edema pulmonar agudo es un efecto adverso infrecuente y potencialmente grave. Desde 1968, se han reportado 50 casos clínicos en la literatura. En este artículo presentamos el caso clínico de una mujer de 70 años atendida en el Hospital Santiago Oriente quien, una hora posterior a la ingesta de hidroclorotiazida, presenta disnea aguda progresiva. El estudio clínico y radiológico es compatible con edema pulmonar agudo no cardiogénico.

Palabras clave: Edema pulmonar agudo, hidroclorotiazida, reacción adversa a medicamentos, unidad de cuidados intensivos.

\section{Introducción}

El edema agudo de pulmón (EPA) es un cuadro frecuentemente asociado a patología cardíaca. Dentro de las causas no cardiogénicas destacan: sepsis, tromboembolismo pulmonar y pancreatitis. Los fármacos representan menos del $3 \%$ de las etiologías, siendo los más frecuentemente reportados los opioides (principalmente la morfina) y el uso de salicilatos.

En Chile, la hidroclorotiazida se utiliza ampliamente como terapia de primera línea en la hipertensión esencial por su bajo costo, fácil disponibilidad y efectividad demostrada. Entre los efectos adversos más conocidos destacan: trastornos hidroelectrolíticos, hiperuricemia, dislipidemia y azotemia. El edema pulmonar agudo es un efecto adverso infrecuente y potencialmente grave. Desde 1968, se han reportado 50 casos clínicos. La etiopatogenia se desconoce.

En este artículo presentamos un caso clínico compatible con edema pulmonar agudo por uso de hidroclorotiazida atendido en el Hospital Santiago Oriente.

\section{Descripción del caso}

Se trata de una paciente mujer de 70 años con antecedente de hipotiroidismo, en tratamiento

\footnotetext{
* Interna de Medicina Universidad de Chile.

** Instituto Nacional del Tórax.

***Hospital Santiago Oriente.
} 
con Levotiroxina (100 $\mu \mathrm{g} /$ día) y trastorno de la conducción cardíaca no especificado, en control en Cardiología, sin farmacoterapia. Sin antecedentes de alergias, consumo de tabaco ni otras sustancias. Se le diagnostica hipertensión arterial esencial en atención primaria y se indica tratamiento con hidroclorotiazida en dosis $50 \mathrm{mg}$ al día. Alrededor de una hora después de ingerir la primera dosis presenta sensación de calor, dificultad respiratoria progresiva, diaforesis y calofríos.

Consulta el mismo día en el Servicio de Urgencias donde se constata que estaba afebril, con frecuencia cardíaca de 105 ciclos por minuto, polipneica y con discreta hipertensión arterial (156/65 mmHg) con saturación de 74,7\% medida por oximetría de pulso, respirando aire ambiental. $\mathrm{Al}$ examen físico general se encuentra vigil y orientada, bien perfundida y con las venas yugulares no ingurgitadas a $45^{\circ}$. A la auscultación pulmonar se pesquisa disminución de murmullo pulmonar en ambos campos asociado a crépitos bilaterales. Al examen cardíaco su ritmo era regular y sin soplos. Tampoco presentaba edema de extremidades inferiores.

Dentro de los exámenes de laboratorio en el
Servicio de Urgencias destaca: hemoglobina $15,7 \mathrm{~g} / \mathrm{dL}$ (VN: 12,0-16,9 g/dL), Leucocitos $6.500 / \mathrm{mm}^{3}$, (VN: $5.000-10.000 / \mathrm{mm}^{3}$ ), Plaquetas $206.000 / \mathrm{mm}^{3}$ (VN: $150.000-400.000 / \mathrm{mm}^{3}$ ), velocidad de eritrosedimentación (VHS) $20 \mathrm{~mm} / \mathrm{h}$ (VN: $1-20 \mathrm{~mm} / \mathrm{h}$ ), proteína $\mathrm{C}$ reactiva (PCR) 0,57 $\mathrm{mg} / \mathrm{dL}(\mathrm{VN}:<1,0 \mathrm{mg} / \mathrm{dL}), \mathrm{PaO}_{2} 72 \mathrm{mmHg}\left(\mathrm{PaO}_{2} /\right.$ $\mathrm{FiO}_{2}=181 \mathrm{mmHg}$ ), Creatinina $0,96 \mathrm{mg} / \mathrm{dL}(\mathrm{VN}$ : 0,6-1,1 mg/dL), nitrógeno ureico sanguíneo 21,5 $\mathrm{mg} / \mathrm{dl}$ (VN: $8,0-20,0 \mathrm{mg} / \mathrm{dL}$ ), Lactato $50,9 \mathrm{mg} /$ $\mathrm{dL}(\mathrm{VN}:<11,3 \mathrm{mg} / \mathrm{dL}$ ), péptido natriuretico tipo B (BNP) $475 \mathrm{pg} / \mathrm{mL}(\mathrm{VN}:<125 \mathrm{pg} / \mathrm{dL})$, Troponina I $0,015 \mathrm{ng} / \mathrm{ml}(\mathrm{VN}:<0,04), \mathrm{CK}-\mathrm{MB}: 2 \mathrm{U} / \mathrm{L}$ (VN:0,6-24,9 U/L), CK total $57 \mathrm{U} / 1$ (VN: 24,0$170,0 \mathrm{U} / \mathrm{L})$, electrolitos plasmáticos y función hepática en rangos normales. El electrocardiograma muestra taquicardia sinusal de 109 ciclos por minuto e imagen de bloqueo de rama izquierda.

El estudio con radiografía simple de tórax, muestra opacidades bilaterales. Se realiza angiotomografia computada de tórax, que muestra opacidades pulmonares bilaterales con patrón "en vidrio esmerilado", sin imágenes de trombos en las arterias pulmonares, sin condensaciones ni derrames pleurales (Figura 1).
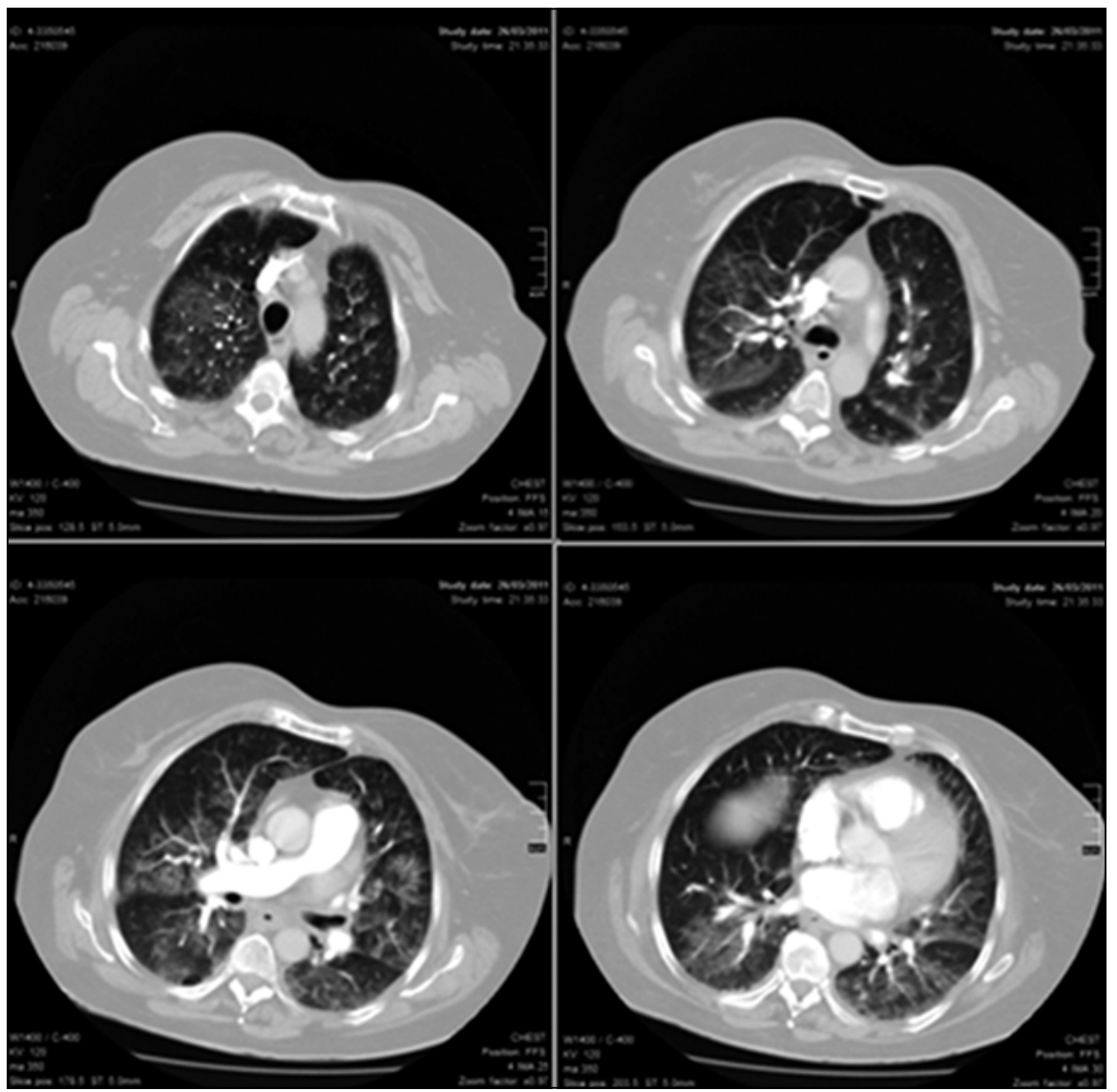

Figura 1. Angio-TAC de tórax al ingreso (ver descripción en el texto). 
El ecocardiograma transtorácico muestra cavidades ventriculares de tamaño normal, hipertrofia ventricular izquierda, movimiento paradojal septal, fracción de eyección de $61 \%$, presión sistólica de arteria pulmonar de $36 \mathrm{mmHg}$ y disfunción diastólica grado I, sin áreas de hipocinesia ni acinesia.

La paciente es trasladada a la unidad de cuidados intensivos (UCI). Ingresa a dicha unidad hemodinámicamente estable, con necesidad de oxígeno por mascarilla de alto flujo. Con fracción inspirada de oxígeno de $50 \%$, su oximetría de pulso era de $93 \%$. Evoluciona con mejoría clínica espontánea y menor requerimiento de $\mathrm{FiO}_{2}$, sin necesidad de monitorización invasiva, uso de drogas vasoactivas o diuréticos. En su evolución presenta alza térmica de $38,5^{\circ} \mathrm{C}$ única y autolimitada. Dos hemocultivos tomados al ingreso resultaron negativos. En el control de exámenes de laboratorio a las $24 \mathrm{~h}$ las concentraciones de lactato arterial y BNP se normalizaron y las enzimas cardíacas se mantuvieron normales.

Tras $48 \mathrm{~h}$ de permanencia en UCI, se trasladó a unidad de menor complejidad y fue dada de alta en buenas condiciones sin control radiológico.

\section{Discusión y comentarios}

El edema pulmonar por hidroclorotiazida fue reportado por primera vez en 1968 por Steinberg $^{1}$. Desde entonces se han descrito aproximadamente 50 nuevos casos. Es un cuadro que cursa con clínica respiratoria inespecífica y de gravedad variable, describiéndose una mortalidad de aproximadamente $6 \%{ }^{2}$. La mayoría de los casos descritos involucran a pacientes de sexo femenino (aproximadamente un 90\%) entre los 40-50 años de edad.

Varios fármacos se han relacionado con la producción de edema pulmonar agudo, entre ellos: opioides, salicilatos y tocolíticos. Sin embargo, estos agentes, a diferencia de la hidroclorotiazida, desencadenan el cuadro en contexto de intoxicación y no en dosis terapéuticas ${ }^{3}$.

Tal como en el caso descrito, el cuadro se inicia dentro de una a dos horas tras la ingesta del fármaco con dosis entre $25-50 \mathrm{mg}$ (dosis $10 \mathrm{mg}$ también se han visto implicadas) y se puede producir tanto en la primera ingesta como en administraciones subsiguientes, siendo más grave en las recurrencias ${ }^{4,5}$. Se ha descrito reacción cruzada con la clorotiazida ${ }^{6}$, no así con diuréticos de otras familias como la furosemida.

La causa y mecanismo de esta reacción aparentemente idiosincrática se desconoce. Manso et al., y Shieh et al, han propuesto un mecanismo de hipersensibilidad tipo I al evidenciar aumento de eosinófilos en el lavado broncoalveolar, aumento en la activación de basófilos en sangre y niveles de IgE plasmáticos en sus pacientes ${ }^{7,8}$. Sin embargo, los resultados han sido inconsistentes en otros casos. Por otro lado, otros autores proponen un mecanismo de hipersensibilidad tipo III con la producción de IgG anti-hidroclorotiazida y depósito de complejos inmunes en la membrana alveolar asociado a infiltración de polimorfonucleares $^{9,10}$.

La manifestación clínica se caracteriza por el inicio agudo de disnea progresiva, dolor torácico y/o abdominal, cianosis, náuseas, vómitos y diarrea $^{3,4}$. Hasta un tercio de los pacientes puede presentar fiebre de carácter auto limitado ${ }^{10}$. En cuanto a los exámenes de laboratorio, éstos suelen ser normales, si bien se han descrito casos que cursan con leucopenia, trombocitopenia, hemoconcentración y marcadores inflamatorios levemente aumentados ${ }^{4}$. Esta paciente se presentó con disnea aislada, insuficiencia respiratoria, hemograma sin alteraciones y sin aumento significativo de marcadores inflamatorios.

En cuanto al estudio etiológico, debe descartarse un factor cardiogénico dado que éste es el principal causante de edema pulmonar agudo. La paciente, si bien tiene un bloqueo completo de rama izquierda al ECG, tiene enzimas cardíacas dentro de límites normales, ausencia de otros signos electrocardiográficos de isquemia, además de un ecocardiograma transtorácico con función sistólica conservada sin signos de hipocinesia o defectos valvulares que indiquen insuficiencia cardíaca como causa del cuadro. La elevación de los niveles de BNP plasmáticos al ingreso, tiene más bien un carácter inespecífico, coexistiendo múltiples factores que contribuyen a su alza (disnea aguda, hipertensión arterial, hipertrofia ventricular izquierda, vasoconstricción pulmonar) ${ }^{11}$.

Los signos radiográficos descritos en la literatura corresponden a infiltrado alvéolo-intersticiales difusos y bilaterales en la radiografía de tórax y patrón de vidrio esmerilado en la $\mathrm{TAC}^{3,4}$. En el caso de la paciente presentada, tanto la radiografía de tórax inicial como la tomografía muestran patrones compatibles, además de descartar tromboembolismo pulmonar, derrame pleural y condensaciones que pudiesen explicar el cuadro (Figura 1).

No existe terapia específica, siendo el manejo basado en la suspensión inmediata del fármaco, el aporte de oxígeno (tanto en su modalidad invasiva como no invasiva) y el manejo general del edema pulmonar agudo. Se ha estimado que hasta 
un tercio de los pacientes requerirá ventilación mecánica invasiva ${ }^{2}$. No se ha demostrado que el uso de corticoides, antibióticos o terapia broncodilatadora altere la evolución y/o pronóstico del cuadro $^{12}$. Esta paciente respondió clínicamente a la administración de oxígeno por mascarilla de alto flujo y cristaloides endovenosos, sin requerir el aporte de fármacos ni monitorización invasiva.

En cuanto a la duración del cuadro se describe en la literatura un promedio $3,5 \pm 0,6$ días; $\sin$ embargo, las alteraciones de la función pulmonar (aumento del gradiente alvéolo-arterial de oxígeno) pueden persistir por más de 30 días sin implicancia clínica. La resolución radiográfica suele coincidir con la mejoría clínica del paciente. No se han reportado aún secuelas a largo plazo por esta condición ${ }^{12}$.

\section{Bibliografía}

1.- STEINBERG A D. Pulmonary edema following ingestion of hydrochlorothiazide. JAMA 1968; 204: 167-9.

2.- BIRON P, DESSUREAULT J, NAPKE E. Acute allergic interstitial pneumonitis induced by hydrochlorothiazide. Can Med Assoc J 1991; 145: 28-34.

3.- REED C, GLAUSER F. Drug-induced noncardiogenic pulmonary edema. Chest 1991; 100: 1120-4.

4.- KNOWLES S R, WONG G A, RAHIM S A, BINKLEY K, PHILLIPS E J, SHEAR N H. Hydrochlorothiazideinduced noncardiogenic pulmonary edema: an unde- rrecognized yet serious adverse drug reaction. Pharmacotherapy 2005 ; 25: 1258-65.

5.- MAS A, JORDANA R, VALLÉS J, CERVANTES M. Recurrent hydrochlorothiazide-induced pulmonary edema. Intensive Care Med 1998; 24: 363-5.

6.- BOWDEN F. Non-cardiogenic pulmonary edema after ingestion of chlorothiazide. Brit Med J 1989; 298: 605.

7.- MANSO S, HEILI M, FERNÁNDEZ-NIETO B, SASTRE J. Basophil activation in two cases of hydrochlorothiazide-induced noncardiogenic pulmonary edema. Allergy 2010; 65: 135-6.

8.- $\quad$ SHIEH C M, CHEN C H, TAO C W, TSAI J J, PERNG R P. Hydrochlorothiazide-induced pulmonary edema: a case report and literature review. Zhonghua Yi Xue Za Zhi 1995; 50: 495-9.

9.- GOETSCHALCKK K, CEUPPENS J, VAN MIEGHEM W. Hydrochlorothiazide-associated noncardiogenic pulmonary edema and shock: a case report and review of the literature. Acta Cardiol 2007; 62: 215-20.

10.- BERNAL C, PATARCA R. Hydrochlorothiazideinduced pulmonary edema and associated immunologic changes. An Pharmacother 1999; 33: 172-4.

11- NAYER J, AGGARWAL P, GALWANKAR S. Utility of point-of-care testing of natriuretic peptides (brain natriuretic peptide and $n$-terminal pro-brain natriuretic peptide) in the emergency department. Int J Chron Obstruct Pulmon Dis 2014; 9: 155-62.

12.- ANDRESEN M, GONZÁLEZ A, ESPINO A, MERCADO M, REQUEIRA T, DOUGNAC A. Thiazide induced acute pulmonary edema: report of one case. Rev Med Chile 2007; 135: 496-500.

Correspondencia a:

Dr. Mauricio Salinas F.

Servicio de Medicina

Instituto Nacional del Tórax

José M. Infante 717 3er piso

Email:mrsf24@hotmail.com 